\title{
Elevated liver function tests in a patient on palbociclib and fulvestrant
}

\author{
Beatrice Alvarado Roberts, $\mathrm{MD},{ }^{\mathrm{a}}$ Mohammed Ibrahim, $\mathrm{RPH},{ }^{\mathrm{b}}$ and Elizabeth Stone, $\mathrm{MD}^{\mathrm{c}}$ \\ Departments of anternal Medicine, ${ }^{\mathrm{b}}$ Oncology Pharmacy, and ${ }^{\mathrm{c} H e m a t o l o g y / O n c o l o g y, ~ C l e v e l a n d ~ C l i n i c ~ F l o r i d a, ~ W e s t o n, ~ F l o r i d a ~}$
}

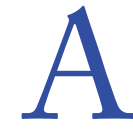

bout $12.4 \%$ of women in the United States will be diagnosed with breast cancer at some point in their lifetime. ${ }^{1} \mathrm{~A}$ percentage of these women will develop metastatic disease and are estimated to have a 5 -year survival rate of $22 \% .^{2}$ There have been meaningful improvements in survival because of earlier detection and more effective systemic therapies. Patients with hormone-sensitive disease often respond to endocrine therapy, and this frequently represents front-line treatment for these patients, resulting in palliation of symptoms while maintaining quality of life.

However, endocrine resistance inevitably occurs, and a great deal of research has been focused on developing strategies to combat resistance. One mechanism of endocrine resistance is though the Cyclin-dependent kinases 4 and 6 (CDK4/6) complexes. Among the most promising of the strategies to prevent resistance are the CDK4/6 inhibitors. There are now 3 approved CDK4/6 inhibitor drugs that can be used in combination with endocrine therapy, 1 of which can also be used as a single agent. When used in combination with endocrine therapy, the use of CDK 4/6 inhibitors has significantly improved progression-free survival (PFS) in patients with hormone-sensitive HER2-negative metastatic breast cancer by inhibiting cellular division and growth. ${ }^{3}$ In postmenopausal women, endocrine therapy plus CDK4/6 inhibitors are the preferred first-line regimen for metastatic disease.

Since the approval of palbociclib by the US Food and Drug Administration in 2015, the most common hematologic lab abnormalities are anemia, leukopenia, neutropenia, and thrombocytopenia. The most common nonhematologic adverse events (AEs) are fatigue, infection, nausea, and stomatitis. Hepatic toxicity has not been commonly observed. We report here the case of a 57-year-old woman on palbociclib and fulvestrant who developed signifi- cant elevation of liver function tests after starting palbociclib, suggesting a possible drug-induced liver injury from palbociclib.

\section{Case presentation and summary}

A 57-year-old woman with history of hypothyroidism and hypertension presented in May 2016 with a lump in her right breast and back pain. The lump was biopsied and revealed invasive ductal carcinoma, moderately differentiated, estrogen receptor (ER) positive $100 \%$, progesterone receptor (PR) positive $95 \%$, and HER2 negative. A positron emission tomography (PET)-computed tomography (CT) scan and magnetic resonance imaging showed bone metastasis at several vertebral levels, and the results of a bone biopsy confirmed metastatic adenocarcinoma of breast origin, ER positive $60 \%, \mathrm{PR}$ positive $40 \%$, and HER 2 negative. No liver lesions were seen on imaging, but there was suggestion of fatty liver. She was started on letrozole $2.5 \mathrm{mg}$ daily in July 2016 while undergoing kyphoplasty and subsequent radiation. A restaging PET scan revealed progression of disease on letrozole, with possible new rib lesion and progression in the breast. No liver disease was noted. Therapy was changed to fulvestrant and palbociclib. Fulvestrant was started in March 2017 with standard dosing of $500 \mathrm{mg}$ intramuscular on days 1,15 , and 29 , and then once a month thereafter. Her first cycle of palbociclib was started on April 5, dosed at $125 \mathrm{mg}$ by mouth daily for 21 days, followed by 7 days off, repeated every 28 days (all dates hereinafter fell within 2017, unless otherwise stipulated).

Labs checked on April 28 and May 26 were unremarkable. A restaging CT scan of the chest, abdomen, and pelvis was done on June 21 after completion of 3 cycles of fulvestrant and palbociclib. There was no evidence of liver metastases, only the fatty infiltration of the liver that had been seen previously.

\footnotetext{
Accepted for publication November 12, 2018. Correspondence: Beatrice Alvarado Roberts, MD; alvarab@ccf.org. Disclosures: The authors report no disclosures or conflicts of interest. JCSO 2018;16(6):e277-e279. (02018 Frontline Medical Communications. doi: https://doi.org/10.12788/jcso.0437
} 
On June 23, 2017, lab results showed a transaminitis with an alanine aminotransferase (ALT) level of $446 \mathrm{IU} / \mathrm{L}$ (reference range 10-33 IU/L) and aspartate aminotransferase (AST) level of $183 \mathrm{IU} / \mathrm{L}$ (reference range 0-32 IU/L).

The patient's liver enzyme levels continued to increase and peaked on July 3 at ALT $>700$ IU/L and AST at 421 IU/L. Her total bilirubin and alkaline phosphatase levels remained within normal limits. She had received her final dose of fulvestrant on May 31 and had taken her last dose of palbociclib on June 20, 2017. She had no history of elevated liver enzymes or liver disease, although the initial PET scan done at diagnosis had suggested hepatic steatosis. She said she had not recently used antibiotics, alcohol, or over-the-counter medications or supplements. There was no family history of liver problems, inflammatory bowel disease, or gastrointestinal malignancy. The only other medications she had taken recently were denosumab, levothyroxine for hypothyroidism, and amlodipine for hypertension. She was seen by hepatology for evaluation of acute hepatitis. Other etiologies for her elevated liver enzymes were ruled out, and she was diagnosed with a drug-induced liver injury from one of her anticancer medications. Her treatments with fulvestrant and palbociclib were held, and the results of her liver function tests normalized by September 2017.

Fulvestrant was restarted on August 24, and her lab results remained normal through November of that year, when restaging scans showed progression with new axillary adenopathy suspicious for metastasis. Imaging also showed a 1.6- $\mathrm{cm}$ hepatic lesion suggestive of a focal area of fat deposition or atypical hemangioma without definitive evidence of metastasis. Follow-up imaging was recommended. She was therefore rechallenged with palbociclib at a reduced dose of $100 \mathrm{mg}$ by mouth daily and received the first dose on November 30. On December 8, repeat labs again showed elevated liver function tests (ALT, $285 \mathrm{IU} / \mathrm{L}$; AST, 112 IU/L). Treatment with palbociclib was discontinued on December 10. Because the patient was not able to tolerate palbociclib, and fulvestrant alone was not controlling the disease, she was started on an alternate endocrine therapy with tamoxifen on December 26. The patient's liver function tests normalized again by January 2018 .

\section{Discussion}

The use of targeted therapies has changed the landscape of oncologic treatments. Several studies have evaluated the safety and efficacy of palbociclib in combination with endocrine therapy. The Palbociclib Ongoing Trials in the Management of Breast Cancer (PALOMA)-1 study, an open-label, randomized, phase-2 trial involving patients with newly diagnosed metastatic hormone sensitive HER2-negative breast cancer, demonstrated that palbociclib in combination with letrozole was associated with significantly longer PFS than letrozole alone. ${ }^{4}$ These results were later confirmed in the larger PALOMA-2 study, a randomized, double-blind, phase- 3 trial that evaluated 666 postmenopausal patients with no prior systemic therapy. In that study, median PFS for the palbociclib-letrozole group was 24.8 months, compared with 14.5 months for the letrozole-alone group (hazard ratio [HR] for disease progression or death, 0.58 [0.46-0.72], $P<.001) .{ }^{5}$ The most recent PALOMA-3 study, a phase-3 trial involving 521 patients with advanced hormone receptor-positive, HER2-negative breast cancer that had progressed during initial endocrine therapy, evaluated the efficacy of combined palbociclib and fulvestrant in a randomized, double-blind, placebo-controlled, parallel-group trial. The result was that the palbociclib-fulvestrant combination resulted in longer median PFS of 9.2 months, compared with 3.8 months with fulvestrant alone $(P<.001) .^{6}$

These trials also monitored the number of AEs as secondary aims. The most commonly reported AEs in the PALOMA trials for those patients in the palbociclib group were hematologic, with neutropenia being the most common, followed by leukopenia, anemia, and thrombocytopenia. The most common nonhematologic AEs reported in the palbociclib-fulvestrant group were fatigue, nausea, and headache. Elevated liver function tests were a rare but reported $\mathrm{AE}$ in $7.2 \%$ of the palbociclib-treated patients in the PALOMA-1 study. ${ }^{7}$ In the PALOMA-2 study, ALT and AST elevations were reported as AEs (all grades) in $9.9 \%$ and $9.7 \%$ of palbociclib-treated patients, respectively. ${ }^{5}$ In the PALOMA-3 study, there was 1 fatal serious $\mathrm{AE}$ of hepatic failure with grade 5 disease progression in the palbociclib group; however, the patient's medical history included progressive liver metastasis and disease progression. ${ }^{6}$ A pooled safety analysis conducted across all PALOMA studies demonstrated that grade 3/4 AST and ALT elevations occurred in $3.3 \%$ and $2.3 \%$ of palbociclibtreated patients, respectively, again highlighting a reported but rare occurrence. ${ }^{8}$

The patient described in the present case report started on combination fulvestrant and palbociclib after her disease showed progression on letrozole. She developed an increase in transaminases after completing 3 cycles of palbociclib. Liver function tests increased nearly 12 weeks after beginning her first cycle of the CDK 4/6 inhibitor. Staging scans of the patient demonstrated fatty liver. It is not known if her fatty liver contributed to her transminitis; however, her baseline labs showed normal liver function tests, and they did not increase until after therapy with fulvestrant-palbociclib was started. It might have been that her fatty liver caused her to be at higher risk of transaminitis with administration of palbociclib, although we cannot be certain. Her lab results remained normal while she was on fulvestrant alone, and the liver function test results increased only after palbociclib was started, making this drug the more likely culprit. 
Both events of increased liver enzymes occurred within a week of the last palbociclib dose; however, we note that hepatotoxicity developed at a faster rate when the patient was rechallenged with palbociclib at a lower dose, with elevated liver function tests increasing 1 week after restarting treatment as opposed to the first episode that occurred after 3 cycles of the palbociclib. After discontinuation of the medication, liver function tests again normalized, suggesting that palbociclib was most likely the causative agent. In addition, the degree of elevated liver enzymes was less severe on re-exposure at the lower dose of $100 \mathrm{mg}$, which raises the possibility that there could be a dose-dependent association between palbociclib and hepatotoxicity. There have been few case reports of increased liver enzymes associated with palbociclib, and it is only recently that this association has been more recognized. A meta-analysis by Zaw and colleagues has demonstrated that CDK 4/6 inhibitor-based regimens are associated with a higher risk of elevated AST and ALT; however, their relation with dose dependence was not described. In particular, they found that CDK 4/6 inhibitors increased the risk of high-grade,

\section{References}

1. Howlader N, Noone AM, Krapcho M, et al. SEER cancer statistics review, 1975-2014. Bethesda, MD: National Cancer Institute; 2017. https://seer.cancer.gov/csr/1975_2014/. Accessed April 3, 2018.

2. American Cancer Society. Breast cancer survival rates. https://www. cancer.org/cancer/breast-cancer/understanding-a-breast-cancerdiagnosis/breast-cancer-survival-rates.html. Accessed April 3, 2018.

3. Wolff AC. CDK4 and CDK6 inhibition in breast cancer - a new standard. N Engl J Med. 2016; 375(20):1993-1994.

4. Finn RS, Crown JP, Lang I, et al. The cyclin-dependent kinase 4/6 inhibitor palbociclib in combination with letrozole versus letrozole alone as first-line treatment of oestrogen receptor-positive, HER2negative, advanced breast cancer (PALOMA-1/TRIO-18): a randomized phase 2 study. Lancet Oncol. 2015;16(1):25-35.

5. Finn RS, Martin M, Rugo, HS et. al. Palbociclib and letrozole in advanced breast cancer. New Engl J Med. 2016;375:1925-1936

6. Cristofanilli M, Turner NC, Bondarenko I, et al. Fulvestrant plus pal- elevated ALT with a relative risk of 4.33 (95\% confidence interval, 2.15-8.71; $P<.0001$ ). The meta-analysis also included other CDK 4/6 inhibitors such as abemaciclib and ribociclib, which have been more commonly associated with liver toxicity than palbociclib has. ${ }^{9}$ Our case report highlights the specific association between palbociclib and elevated liver enzymes.

In conclusion, this case report illustrates that our patient's elevated liver enzymes were likely related to palbociclib. This is further supported by the fact that this AE occurred twice, both times after palbociclib exposure. In each instance, liver enzymes normalized after discontinuation of palbociclib. One cannot entirely rule out that fulvestrant might have been the culprit medication, but the patient's normal hepatic panel for several months after starting fulvestrant suggests that is less likely. This case report is indicative of an uncommon complication in the treatment of metastatic breast cancer, one that is starting to gain more recognition, and we must think of palbociclib as a possible cause of drug-induced liver injury when targeted CDK 4/6-based regimens are used. bociclib versus fulvestrant plus placebo for treatment of hormonereceptor-positive, HER2-negative metastatic breast cancer that progressed on previous endocrine therapy (PALOMA-3): final analysis of the multicentre, double-blind, phase 3 randomized controlled trial. Lancet Oncol. 2016;17(4):425-439.

7. Turner NC, Ro J, André F, et al. Palbociclib in hormone-receptor-positive advanced breast cancer. N Engl J Med. 2015;373(3):209-219.

8. Dieras V, Rugo HS, Schnell P, et al. Long-term pooled safety analysis of palbociclib in combination with endocrine therapy for $\mathrm{HR}+/ \mathrm{HR}-$ advanced breast cancer [published online July 18, 2018]. Natl Cancer Inst. 2018;111.

9. Zaw M, Thein KZ, Tun A, et al. A systematic review and meta-analysis of randomized controlled trials to evaluate the risk of gastrointestinal and hepatic toxicities in patients with hormone receptor positive HER2-negative breast cancer treated with CKD 4/6 inhibitors. J Clin Oncol. 2017;35(suppl 31):209. 